\title{
Estilos de aprendizaje: La experiencia de la Escuela Preparatoria Técnica Médica en la Universidad Autónoma de Nuevo León, México
}

\author{
Learning Styles: The Experience of the Medical School at the University of Nuevo León, Mexico \\ Estilos de aprendizagem: A experiência de uma faculdade de educação técnica médica em \\ uma universidade de Nuevo Leon, México
}

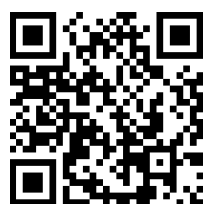

Pedro César Cantú-Martínez'

Universidad Autónoma de Nuevo León

Monterrey, Nuevo León, México

cantup@hotmail.com

iD http://orcid.org/0000-0001-8924-5343

Julia Magdalena Rojas-Márquez²

Universidad Autónoma de Nuevo León

Monterrey, Nuevo León, México

malena.rojas@hotmail.com

http://orcid.org/0000-0002-5172-5955

Recibido • Received • Recebido: 19 / 07 / 2016

Corregido • Revised • Revisado: $10 / 12$ / 2017

Aceptado • Accepted • Aprovado: 25/ 01 / 2018

\begin{abstract}
Resumen: El objetivo del presente artículo está encaminado a precisar el estilo de aprendizaje del estudiantado de la Escuela Preparatoria Técnica Médica en la Universidad Autónoma de Nuevo León, México. En esta investigación participaron 333 estudiantes entre mujeres y varones, quienes respondieron el cuestionario de Honey-Alonso para determinar sus estilos de aprendizaje. Los resultados emanados manifiestan que las personas participantes tienden a poseer un estilo de aprendizaje reflexivo y pragmático, que es acorde con la orientación de su preparación profesional, y es coincidente con otros estudios similares.
\end{abstract}

Palabras claves: Estilos de aprendizaje; estudiantes; técnicos; preparatoria; medicina.

\footnotetext{
${ }^{1}$ Doctorado en Ciencias Biológicas por la Universidad Autónoma de Nuevo León (UANL) en México. Profesor de la UANL, adscrito a la Facultad de Ciencias Biológicas. Miembro del Sistema Nacional de Investigadores - CONACYT: Nivel 1. Miembro del Cuerpo Académico de Ciencias Exactas y Desarrollo Humano UANL-CA-181 con nivel consolidado. Su línea de investigación es desarrollo humano, calidad de vida y sustentabilidad.

${ }^{2}$ Licenciada en Pedagogía, Maestra de la Universidad Autónoma de Nuevo León inscrita a la Escuela y Preparatoria Técnica Médica. Su línea de investigación es educación.
} 
doi: http://dx.doi.org/10.15359/ree.22-2.3

URL: http://www.una.ac.cr/educare

CORREO: educare@una.cr

\begin{abstract}
This article aims to clarify the learning style of students in the Medical School at the Autonomous University of Nuevo Leon (Mexico). In this research participated 333 students, men and women. They responded to a Honey-Alonso questionnaire to determine their learning styles. The results showed that the participants tend to possess a reflective and pragmatic learning style, which is consistent with the orientation of their professional preparation, and with other similar studies.
\end{abstract}

Keywords: Learning styles, students, technicians, school, medicine.

Resumo: O objetivo deste artigo destina-se a esclarecer o estilo de aprendizagem de estudantes de uma faculdade de educação técnica médica da Universidade Autônoma de Nuevo Leon (México). Esta pesquisa envolveu 333 estudantes entre homens e mulheres, que responderam ao questionário Honey-Alonso para determinar estilos de aprendizagem. Os resultados encontrados mostram a tendência das pessoas participantes a um estilo de aprendizagem pragmática e de reflexão, o que é consistente com a orientação de sua formação profissional, e também consistente com outros estudos semelhantes.

Palavras chaves: Estilos de aprendizagem; estudantes; técnicos; faculdade; medicina.

\title{
Introducción
}

Se reconoce que la educación ya no es la misma, de acuerdo con González (2011, p. 2), en la"segunda década del siglo XXI las teorías acerca del aprendizaje aumentan, varían, se reajustan a los [diferentes] contextos". Por lo tanto, resulta de sumo interés cómo estas proposiciones pretenden ahondar en el aprendizaje y determinar cómo el estudiantado particularmente asimila los contenidos, de los que son abastecidos por sus docentes (Salas-Cabrera, 2014). Dentro de este conjunto de circunstancias, una de suma importancia la ostentan los estilos de aprendizaje, que es donde el estudiantado asume un papel activo, en relación con sus "preferencias y modos individuales de cómo perciben y procesan la información. Estas preferencias individuales son Ilamadas estilos de aprendizaje" (Romero, Salinas y Montera, 2010, p. 5). Estos después se traducen en aprendizaje revelador, y más tarde se pueden emplear en contextos distintos a los escenarios donde se estudiaron y practicaron, conllevando, así, a un cambio en la conducta (Aguilar y Rivera, 2005; Esguerra y Guerrero, 2010).

Con respecto a los estilos de aprendizaje, existen distintas posturas para lograr evaluar y comprender el aprendizaje en el estudiantado. Estos estilos de aprendizaje se inclinan por modelos cognitivos y de orientación psicológica, los cuales, indistintamente de cómo se aborden, se reconocen como las vías predilectas del alumnado para aprender, sobre las que se puede intervenir tanto intelectual como psicológicamente (Romero et al., 2010). Se sabe que los estilos de aprendizaje presentes en el alumnado pueden ser reformulados, no obstante su condición de cierta estabilidad en ellos; pues en este proceso intervienen los rasgos de carácter cognitivo, afectivo y fisiológico del estudiantado (Aragón y Jiménez, 2009; Freiberg y Fernández, 2015; Navarro, 2008). 
Particularmente, en la presente investigación llevamos a cabo la tipificación de los estilos de aprendizaje preponderantes, de acuerdo con aquellos desarrollados por Honey y Mumford en 1986, los cuales se sustentan en el trabajo de Kolb (llevado a cabo en 1984 y citado por Freiberg y Fernández, 2015). Estos hacen referencia a cuatro estilos de aprendizaje que son precisados como activo, reflexivo, pragmático y teórico (Cuestionario CHAEA). Salas-Cabrera (2014, p. 162) lo puntualiza sucintamente de la siguiente manera:

El estilo de aprendizaje activo es aquel en el cual la persona improvisa, arriesga, descubre y es espontánea; además, inventa, trata de vivir las experiencias y se mantiene participando de manera dinámica en la clase. Por su parte, el estilo de aprendizaje reflexivo está caracterizado porque la persona es receptiva, analítica y observadora. Los individuos con características de aprendizaje pragmático presentan un estilo caracterizado por ser rápidos, decididos, planificadores, concretos, con objetivos definidos, seguros. Y, finalmente, aquellas personas con un estilo de aprendizaje teórico se caracterizan por ser disciplinadas, sistemáticas, ordenadas, sintéticas, razonadoras, pensadoras, perfeccionistas, buscadoras de modelos teóricos que faciliten la forma de aprender.

Esencialmente, al hacer las indagaciones al respecto (Adán, 2004; Aguilar y Rivera, 2005; Aragón y Jiménez, 2009; Canalejas et al., 2005; Freiberg y Fernández, 2015; García y Ortega, 2012; Ordóñez, Rosety-Rodríguez y Rosety-Plaza, 2003; Romero et al., 2010; Salas-Cabrera, 2014), se ha evidenciado que de manera genérica todos los individuos aprenden de distintas formas, por lo cual se hace pertinente investigar cuáles son los estilos de aprendizaje que muestra el estudiantado de una escuela y preparatoria técnica médica.

\section{Metodología}

La presente investigación fue de carácter descriptivo, comparativo y transversal. Se llevó a cabo de 2013 a 2014, cuyo propósito ha sido determinar el estilo de aprendizaje que ostentan 333 estudiantes de ambos géneros del turno matutino de una escuela y preparatoria técnica médica de la Universidad Autónoma de Nuevo León (Nuevo León, México), de primer semestre, que oscilan entre las edades de 14 a 16 años. La finalidad fue mejorar la trayectoria y los resultados académicos del estudiantado. Para determinar los estilos de aprendizaje se utilizó el cuestionario CHAEA (Honey-Alonso citado por Alonso, Gallego y Honey, 2002), el cual consta de 80 items con dos opciones por respuesta; si está más de acuerdo con el ítem se señala con un (+) y si se está más en desacuerdo se indica con un (-). Cuenta con un puntaje máximo de 20 para la evaluación de cada estilo de aprendizaje. El estilo de aprendizaje que muestre mayor puntaje será el estilo preponderante en el participante (Salas-Cabrera, 2014). Posteriormente a la aplicación y catalogación de las encuestas contestadas, se procedió a la creación de la base de datos para ser analizada con el programa SPSS. 
doi: http://dx.doi.org/10.15359/ree.22-2.3

URL: http://www.una.ac.cr/educare

CORREO: educare@una.cr

\section{Resultados}

De los 333 estudiantes participantes que se encuestaron $29.4 \%$ ( $f=98$ ) contaba con 14 años, 33\% ( $f=110)$ mostraba 15 años y 37.5\% $(f=125)$ evidenciaron una edad de 16 años. El $58.8 \%(f=196)$ correspondió al género femenino, de las cuales $27.6 \%(f=54)$ ostentaba 14 años, $30.6 \%(f=60)$ contaba con 15 años y $41.8 \%(f=82)$ tenía 16 años. Mientras $41.1 \%(f=137)$ fueron varones, los cuales mostraban $32.1 \%(f=44)$ con una edad de 14 años, $36.5 \%(f=50)$ evidenciaron una edad de 15 años y $31.4 \%$ ( $f=43$ ) revelaron 16 años.

Como se muestra en la Tabla 1 se obtuvieron los valores promedio para los diferentes estilos de aprendizaje, resultando el más preponderante para el total de la población estudiada el estilo "Reflexivo" con una valor promedio y desviación estándar de 13.55 ( \pm 2.763$)$. Semejante situación trasciende cuando se observa que este mismo estilo de aprendizaje, el "Reflexivo", se muestra tanto para mujeres como para hombres, con valores $13.84( \pm 0.197)$ y $13.13( \pm 0.233)$, respectivamente.

Tabla 1: Valores promedio de estilos de aprendizaje

\begin{tabular}{cccc}
\hline Estilo de aprendizaje & $\begin{array}{c}\text { Población total } \\
\text { M (DS) }\end{array}$ & $\begin{array}{c}\text { Población femenina } \\
\text { M (DS) }\end{array}$ & $\begin{array}{c}\text { Población masculina } \\
\text { M (DS) }\end{array}$ \\
\hline Activo & $12.08( \pm 3.309)$ & $12.13( \pm 0.244)$ & $12.01( \pm 0.269)$ \\
Reflexivo & $13.55( \pm 2.763)$ & $13.84( \pm 0.197)$ & $13.13( \pm 0.233)$ \\
Teórico & $12.78( \pm 3.068)$ & $12.77( \pm 0.230)$ & $12.79( \pm 0.243)$ \\
Pragmático & $12.84( \pm 2.880)$ & $12.88( \pm 0.198)$ & $12.77( \pm 0.259)$ \\
$n$ & 333 & 196 & 137 \\
\hline
\end{tabular}

M (DS), Media ( \pm Desviación estándar); n: número de participantes.

Nota: Elaboración propia.

En relación con los estilos de aprendizaje presentes, en las mujeres el de mayor frecuencia fue el "Reflexivo" con 31.1\% ( $f=61)$, siguiéndole el "Activo", "Teórico" y "Pragmático", con $18.4 \%(f=36), 15.8 \%(f=31)$ y $12.8 \%(f=25)$ respectivamente $y$, en menor frecuencia, aquellos que manifestaron contar con dos o más estilos de aprendizajes. En cuanto a los varones, 25.5\% ( $\mathrm{f}=35$ ) ostentaron un estilo de aprendizaje "Reflexivo", continuando el "Pragmático", "Activo" y "Teórico", con recuentos de $17.5 \%(f=24), 15.3 \%(f=21)$ y $13.9 \%(f=19)$ correspondientemente $y$, en menor reiteración, aquellos que revelaron contar con dos o más estilos de aprendizajes. En el estudio no aparece asociación significativa entre los estilos de aprendizaje y el género (valor de p > 0.05).

En relación con el análisis por grupos, se obtuvo que en el $80 \%(f=8)$ prevalecía un estilo de aprendizaje "Reflexivo"; mientras que con un 10\% $(\mathrm{f}=1)$ imperaba tanto el estilo "Teórico" como el "Pragmático". Por otra parte, se encontraron diferencias significativas entre los valores 
promedio cuantificados tanto para los estilos "Reflexivo", como se observa entre el Grupo 05 y Grupo 08 con una confiabilidad del 95\%, y en el "Pragmático", como se advierte entre el Grupo 03 y Grupo 08, y Grupo 10 y el Grupo 08 con una confiabilidad del 99\% respectivamente. No así, para los estilos Activo y Teórico (ver tabla 2).

Tabla 2: Valores promedio de estilos de aprendizaje por grupo

\begin{tabular}{cccccc}
\hline Grupo & $\mathrm{n}$ & $\begin{array}{c}\text { Activo } \\
\mathrm{M}(\mathrm{DS})\end{array}$ & $\begin{array}{c}\text { Reflexivo } \\
\mathrm{M}(\mathrm{DS})\end{array}$ & $\begin{array}{c}\text { Teórico } \\
\mathrm{M}(\mathrm{DS})\end{array}$ & $\begin{array}{c}\text { Pragmático } \\
\mathrm{M}(\mathrm{DS})\end{array}$ \\
\hline $\mathrm{G} 01$ & 25 & $12.92( \pm 0.55)$ & $13.04( \pm 0.49)$ & $13.72( \pm 0.68)$ & $13.60( \pm 0.45)$ \\
G02 & 38 & $13.03( \pm 0.52)$ & $13.79( \pm 0.50)$ & $12.74( \pm 0.49)$ & $12.79( \pm 0.46)$ \\
G03 & 13 & $11.92( \pm 0.67)$ & $14.38( \pm 0.61)$ & $13.85( \pm 0.49)$ & $14.62( \pm 0.57)^{\mathrm{a}}$ \\
G04 & 39 & $12.18( \pm 0.44)$ & $12.87( \pm 0.41)$ & $11.85( \pm 0.45)$ & $12.79( \pm 0.49)$ \\
G05 & 46 & $11.22( \pm 0.53)$ & $14.65( \pm 0.36)^{\mathrm{a}}$ & $12.80( \pm 0.43)$ & $12.89 \pm(0.41)$ \\
G06 & 49 & $12.41( \pm 0.52)$ & $13.37( \pm 0.37)$ & $12.31( \pm 0.43)$ & $12.55( \pm 0.37)$ \\
G07 & 34 & $12.35( \pm 0.53)$ & $13.65( \pm 0.47)$ & $13.59( \pm 0.50)$ & $12.58( \pm 0.45)$ \\
G08 & 39 & $10.72( \pm 0.57)$ & $12.49( \pm 0.42)^{\mathrm{a}}$ & $12.41( \pm 0.45)$ & $11.38( \pm 0.42)^{\mathrm{a} . \mathrm{b}}$ \\
G09 & 26 & $11.62( \pm 0.56)$ & $13.77( \pm 0.55)$ & $12.62( \pm 0.62)$ & $12.92( \pm 0.64)$ \\
G10 & 24 & $12.96( \pm 0.66)$ & $13.96( \pm 0.67)$ & $13.33( \pm 0.82)$ & $13.92( \pm 0.72)^{\mathrm{b}}$ \\
\hline Valor $p$ & & $\mathrm{Ns}$ & $\mathrm{p} \leq 0.05$ & $\mathrm{Ns}$ & $\mathrm{p} \leq 0.01$ \\
\hline
\end{tabular}

n, número de participantes; M (DS), Media ( \pm Desviación estándar); Ns, No significativa.

Nota: Elaboración propia.

\section{Discusión}

En el ámbito educativo los estilos de aprendizaje florecen desde hace más de cuatro décadas y se han ostentado en el contexto educativo como una noción para examinar las diferencias específicas que surgen entre el estudiantado en lo que atañe a las formas en que un individuo procesa la información hasta llegar a su apropiamiento, por lo tanto, el análisis de los estilos de aprendizaje ha revestido mucho interés desde un contexto teórico y aplicado (Gamboa, Briceño y Camacho, 2015). Como se ha podido observar con los resultados obtenidos en nuestra investigación, el perfil de aprendizaje más preponderante en los estudiantes de una escuela y preparatoria técnica médica es el "Reflexivo", tanto para mujeres como para varones. Este estilo de aprendizaje se caracteriza en personas que prefieren registrar la experiencia 
doi: http://dx.doi.org/10.15359/ree.22-2.3

URL: http://www.una.ac.cr/educare

CORREO: educare@una.cr

mediante acopiar "datos y los analizan detenidamente, son ponderados, concienzudos, receptivos, analíticos y exhaustivos" (Canalejas et al., 2005, p. 84). Y en segunda instancia son Pragmáticos, es decir, que "prefieren la aplicación práctica de las ideas. Son experimentadores, prácticos, directos, eficaces y realistas" (Canalejas et al., 2005, p. 84).

El ser "Reflexivo" y "Pragmático" por parte del estudiantado participante es congruente con la orientación que demarca su preparación profesional técnica médica, donde la aplicación del conocimiento debe ser ostensiblemente preconcebida, razonable y eficiente, lo que se constituye en la base fundamental para actuar con pertinencia en la atención en salud $y$, principalmente, con pacientes en situaciones comprometidas. Asimismo, los resultados de nuestra investigación son similares a los de otros estudios que expresan que el estilo de aprendizaje "Reflexivo" es particularmente mostrado por aquel alumnado que estudia carreras en el área de las ciencias de la salud, como así lo denotan las investigaciones realizadas por Canalejas et al. (2005), Ordóñez et al. (2003) y Salas-Cabrera (2014).

Si bien es cierto que el estilo de aprendizaje y el actuar está prescrito, en gran parte, por cada individuo, donde interviene una adaptación de carácter académico que se configura de acuerdo con numerosos factores socioambientales, este puede verse influido, como todo acto didáctico, por la dinámica proporcionada y estimulada por el personal docente en el aula. Al respecto, debemos recordar que durante el discurrir de una reconstrucción de una experiencia, se puede promover una meditación constante con deliberación, donde se pueden instaurar nexos con conocimientos de índole teórico, práctico y aplicativo (Cardona, 2013).

Por otra parte, Loría-Castellanos, Rivera, Gallardo, Márquez-Ávila y Chavarría-Islas (2007, p. 9) señalan:

La comprensión y estudio de los estilos de aprendizaje proporciona sin dudas, información básica, aunque no suficiente para organizar la teoría y práctica de un proceso de enseñanzaaprendizaje que pretenda tributar al desarrollo de la personalidad [del estudiantado].

Esta última aseveración ha permitido advertir una mayor sensibilización en el profesorado en el área de las ciencias de la salud, particularmente de las instituciones de educación media superior y superior al "considerar al alumno como polo activo del proceso de aprendizaje" (LoríaCastellanos et al., 2007, p. 2); sustentado esto en la reiterada preocupación existente producto de los alcances éticos que conllevará el actuar del estudiantado en el ámbito profesional (Mora, 2005). Particularmente, esto resulta de sumo interés por el hecho de que subsiste, en las disciplinas del área de la salud, la "inclinación a llevar, inmediatamente, el conocimiento alcanzado a la práctica, pues en estas disciplinas la esencia de la enseñanza está determinado por la parte práctica" (Salas-Cabrera, 2014, p. 166) y, si se hace hincapié en nuestro estudio, donde el estudiantado participante se encuentran en la adolescencia, ello retoma considerable relevancia.

6 
Sin duda, el esclarecimiento del estilo de aprendizaje en el estudiantado participante puede favorecer dos líneas conductoras. La primera se relaciona con el mismo estudiantado, al permitirle, a partir de este discernimiento, hacer los ajustes pertinentes para mejorar y asegurar su trayectoria académica; y la segunda, el análisis que se desprende para mejorar en los procesos de enseñanza-aprendizaje, y contribuir al perfeccionamiento de la personalidad y perfil profesional deseado, a lo que lacónicamente Anderson (1995) y Serasin (2006), citados por Gallego (2013, p. 10), señalan al advertir que se ha "demostrado que la relación entre los Estilos de Aprendizaje y los Estilos de Enseñanza es un factor importante en el éxito de los estudiantes".

\section{Referencias}

Adán, M. I. (Julio, 2004). Estilos de aprendizaje y rendimiento académico en las modalidades de bachillerato. I Congreso Internacional de Estilos de Aprendizaje (pp. 1-24). Universidad Nacional de Educación a Distancia, Madrid.

Aguilar, Ú. y Rivera, L. (2005). Los estilos de aprendizaje en el rendimiento académico de los estudiantes de industrias alimentarías del Instituto Superior Tecnológico Publico: Edilberto Rivas Vásquez. Distrito de Cayalti (Tesis de licenciatura). Universidad Nacional Pedro Ruiz Gallo. Lambayeque, Perú. Recuperado de https://es.slideshare.net/lurig6r/tesis-paraoptar-licenciatura-en-educacion-6834948

Alonso, C. M., Gallego, D. J. y Honey, P. (2002). Los estilos de aprendizaje. Procedimientos de diagnóstico y mejora. Bilbao: Ediciones Mensajero.

Aragón, M. y Jiménez, Y. I. (2009). Diagnóstico de los estilos de aprendizaje en los estudiantes: Estrategia docente para elevar la calidad educativa. CPU-e Revista de Investigación Educativa, 9, 1-21. Recuperado de http://www.redalyc.org/articulo.oa?id=283121714002

Canalejas, M., Martínez, M. L., Pineda, M. C., Vera, M. L., Soto, M., Martín, Á. y Cid, M. L. (2005). Estilos de aprendizaje en los estudiantes de enfermería. Educación Médica, 8(2), 83-90. doi: https://doi.org/10.4321/S1575-18132005000200006

Cardona, J. (2013). Epistemología del saber docente. Madrid: Editorial UNED.

Esguerra, G. y Guerrero, P. (2010). Estilos de aprendizaje y rendimiento académico en estudiantes de psicología. Revista Diversitas: Perspectivas en Psicología, 6(1), 97-109. doi: https://doi. org/10.15332/s1794-9998.2010.0001.07

Freiberg, A. y Fernández, M. M. (2015). Estilos de aprendizaje en estudiantes universitarios ingresantes y avanzados de Buenos Aires. Liberabit, 21(1), 71-79. Recuperado de http:// www.scielo.org.pe/pdf/liber/v21n1/a07v21n1.pdf 
doi: http://dx.doi.org/10.15359/ree.22-2.3

URL: http://www.una.ac.cr/educare

CORREO: educare@una.cr

Gallego, D. J. (2013). Ya he diagnosticado el estilo de aprendizaje de mis alumnos y ahora ¿qué hago? Revista de Estilos de Aprendizaje, 11(12), 1-15. Recuperado de http://www2.uned.es/ revistaestilosdeaprendizaje/numero 12/articulos/articulo 1.pdf

Gamboa, M. C., Briceño, J. J. y Camacho, J. P. (2015). Caracterización de estilos de aprendizaje y canales de percepción de estudiantes universitarios. Opción, 31(Especial 3), 509527. Recuperado de http://produccioncientificaluz.org/index.php/opcion/article/ view/20497/20408

García, L. y Ortega, J. C. (2012). Influencia de los estilos de aprendizaje y la autorregulación en el rendimiento escolar de los alumnos del bachillerato a distancia del Estado de México. Revista Mexicana de Bachillerato a Distancia, 4(7), 122-131. Recuperado de http://www. revistas.unam.mx/index.php/rmbd/article/view/45262/40783

González, M. V. (2011). Estilos de aprendizaje: Su influencia para aprender a aprender. Revista Estilos de Aprendizaje, 7(7), 1-13. Recuperado de http://www2.uned.es/ revistaestilosdeaprendizaje/numero 7/articulos//sr 7 articulo 12.pdf

Loría-Castellanos, J., Rivera D. B., Gallardo S. A., Márquez-Ávila, G. y Chavarría-Islas, R., A. (2007). Estilos de aprendizaje de los médicos residentes de un hospital de segundo nivel. Educación Médica Superior, 21(3), 1-13. Recuperado de http://scielo.sld.cu/pdf/ems/v21n3/ ems01307.pdf

Mora, M. (2005). El educador como agente reflexivo en el proceso de formación educativa. Horizontes Educacionales, 10, 71-76. Recuperado de http://www.redalyc.org/ pdf/979/97917573007.pdf

Navarro, M. J. (2008). Cómo diagnosticar y mejorar los estilos de aprendizaje. Almería: Editorial Procompal.

Ordóñez, F.J., Rosety-Rodríguez, M.y Rosety-Plaza, M. (2003). Análisis de los estilos de aprendizaje predominantes entre los estudiantes de ciencias de la salud. Enfermería Global, 2(2), 1-6. Recuperado de http://revistas.um.es/eglobal/article/view/619/645

Romero, L. N., Salinas, V. y Montera, F. J. (2010). Estilos de aprendizaje basados en el modelo de Kolb en la educación virtual. Apertura, 2(1), 1-21. Recuperado de http://www.redalyc.org/ articulo.oa?id=68820841007

Salas-Cabrera, J. (2014). Estilos de aprendizaje en estudiantes de la Escuela de Ciencias del Movimiento Humano y Calidad de Vida, Universidad Nacional, Costa Rica. Revista Electrónica Educare, 18(3), 159-171. doi: https://doi.org/10.15359/ree.18-3.9 